\title{
"Modification, Destruction and Burning": A Research toward the Development Process of Xiamen Dada
}

\author{
Siu Ki Cheung \\ Communication University of China
}

\begin{abstract}
This article reconstructs Xiamen Dada's development process via its artworks, correspondence, and documentary materials. The image analysis and interpretive content analysis have been used in the research toward the materials above. This study fulfilled the lack of the specific materials analysis in the previous studies on Xiamen Dada. It demonstrates a more comprehensive picture of the reaction when Xiamen Dada copes with the social influence. This paper will serve as a foundational source for further cross-disciplinary culture study.
\end{abstract}

Keywords: Xiamen Dada, 85’ New Wave, Chinese Contemporary Art.

\section{The Previous Study about Xiamen Dada}

Fei Da-Wei said that "Xiamen Dada is the first group to abandon crafted painting tradition to consider the art as the concept and work with it in extreme approach." (Fei Da-Wei, 2007) Xiamen Dada is the pioneer of the 85' New Wave or even Chinese contemporary art. But the research to their remaining content is absent. The recent research on Xiamen Dada still focuses on the art criticism field. Lu Ming-Jun described Xiamen Dada as a result of the rethinking of socialist realist art in the art and political view (Lu Ming-Jun,2021) Zhang Si-Qi concluded Huang Yong-Ping's absorption of Wittgenstein's philosophical ideas and Eastern philosophies "Tao" and "Zen" (Zhang Si-Qi, 2019). Shao Tian-Hua sorted out the origin of Xiamen Dada's name and the social background of the founding of the group (Shao Tian-Hua, 2013). Li Sheng-Zhao proposed that the depth of the anti-feudal ideology is what makes Xiamen Dada the most profound and radical criticism and reactionary of the $85^{\prime}$ New Wave (Li Sheng-Zhao, 2010).

However, there are not sufficient materials of Xiamen Dada, which makes it difficult to reconstruct a whole picture of the group. Researches so far have lacked the specific study of Xiamen Dada. A majority of them located Xiamen Dada in the macroscopic perspective of political science, intellectual history, and cultural history, ignoring its dynamics concerning the social environment. The interaction between the mainstream of society and Xiamen Dada is limited in a macroscopic way. Therefore, it is necessary to pay attention to work on artworks, correspondence, and documentary materials. This article will use image analysis and interpretive content analysis to read the content above. Since the Image Analysis of Erwin Panofsky toward the method of composition cannot cover the diverse expression of Xiamen Dada's artworks. This article incorporates a various analysis of conceptual and stylistic expression of works. After this essay, the author plans to conduct further research through the lens of culture studies.

\section{Xiamen Dada: Paradox and Void}

This section focuses on image analysis of four pieces of artworks of Xiamen Dada. "Including all the art groups and artists in the 85' movement, Xiamen Dada and Huang Yong-Ping have the biggest number of academic documents. As an independent artist, Huang Yong-Ping is the biggest contributor of writing and thinking." To research on Xiamen Dada, Huang Yong-Ping is the person who cannot be ignored. In this section, the author picks two artworks of Huang, "A Pistol Can Ring" and "Firecracker Pants". These two works are creative, respectively in the early and later stages of Xiamen Dada's development process. They represent the working features of him in the Xiamen Dada period, the time that he focused on the intersection between daily life and violence, adapting the art form of rebellion.

The research toward the other members except Huang Yong-Ping in Xiamen Dada is absent. Therefore, this article tentatively selects the work of Lin Chun, an important member of Xiamen Dada, "The Man Wrapped in Red Cloth and the Reef and Sea Sky", intents to complete the study of the group's work. Lastly, the author tries to summarize the features of Xiamen Dada, extreme rebellion, pioneering and the diversity of creative form via the study toward the most influential performing art "Modification, Destruction and Burning" which was created in Oct 23rd 1986.

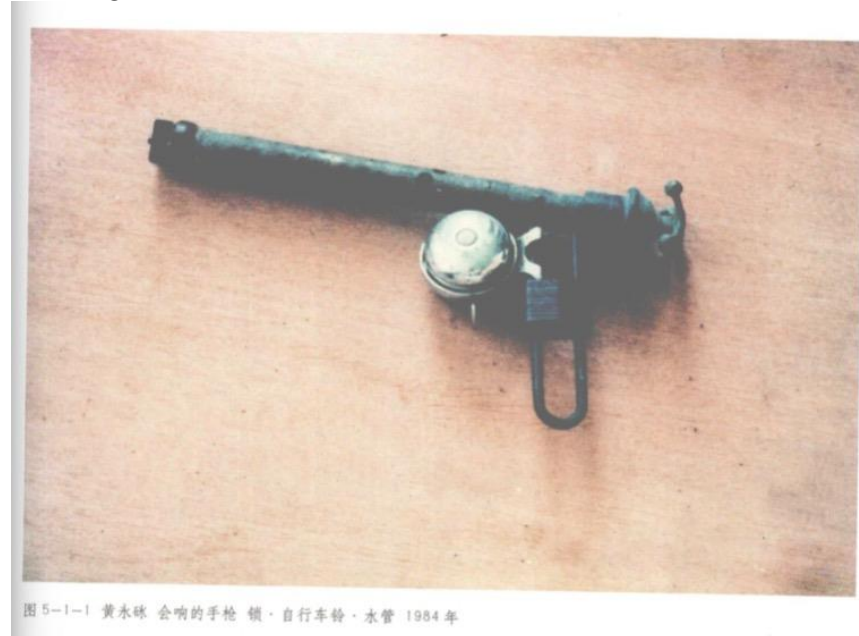

Figure 1: A Pisto Can Ring 


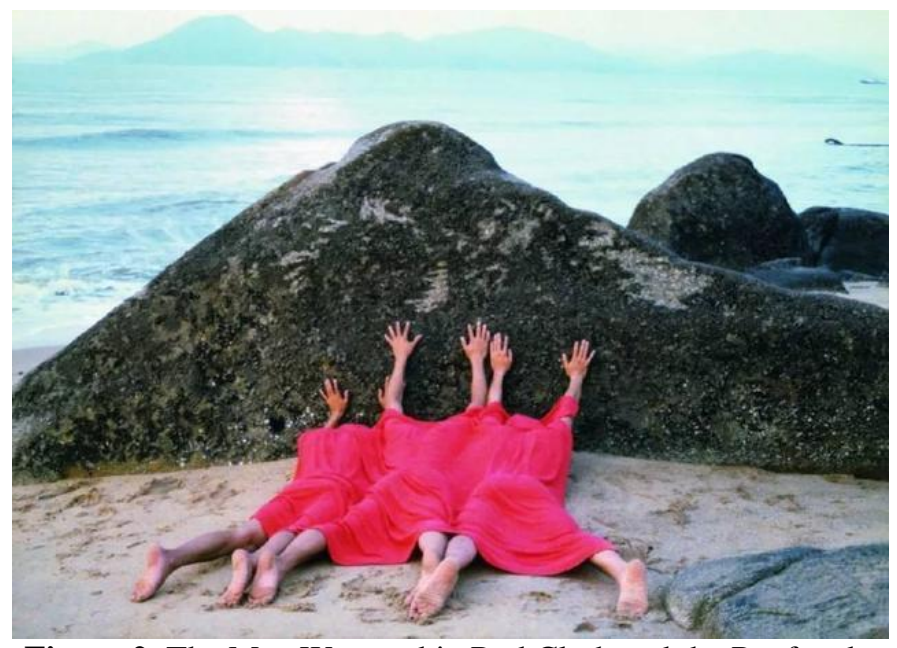

Figure 2: The Man Wrapped in Red Cloth and the Reef and Sea Sky

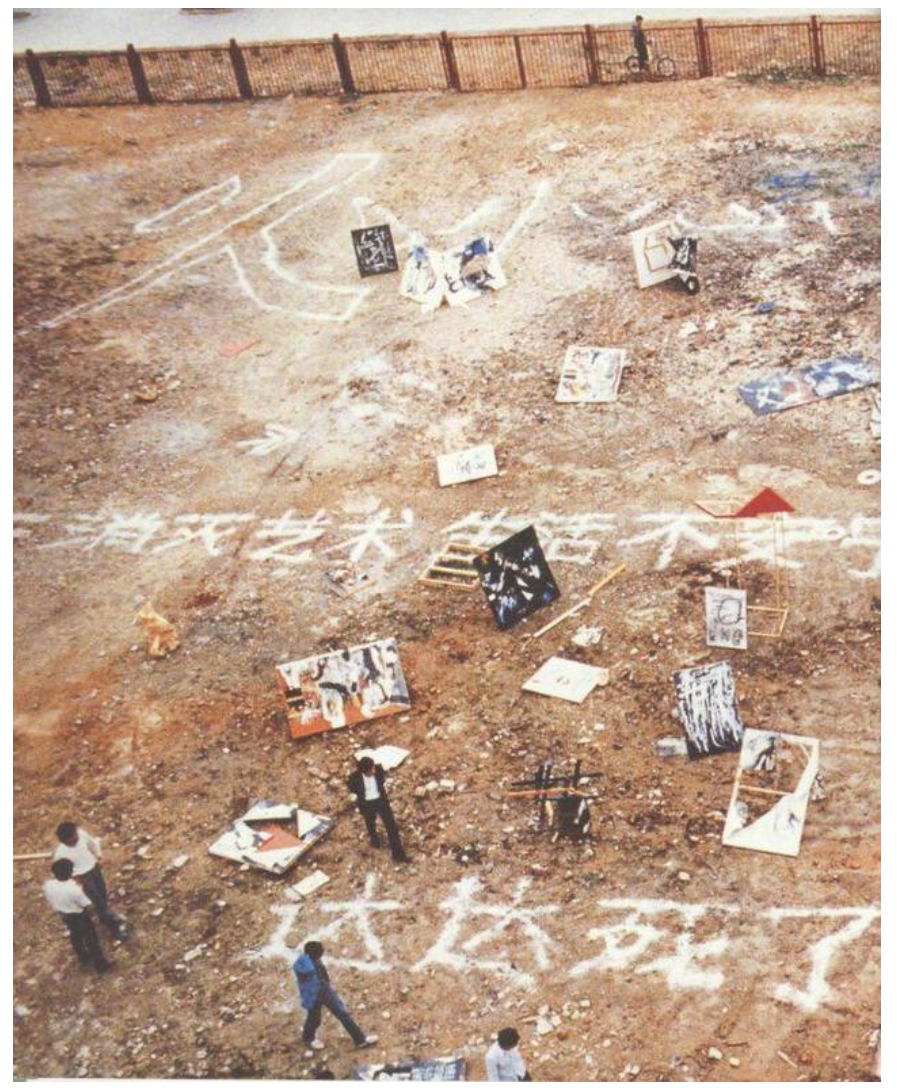

Figure 3: Modification, Destruction and Burning

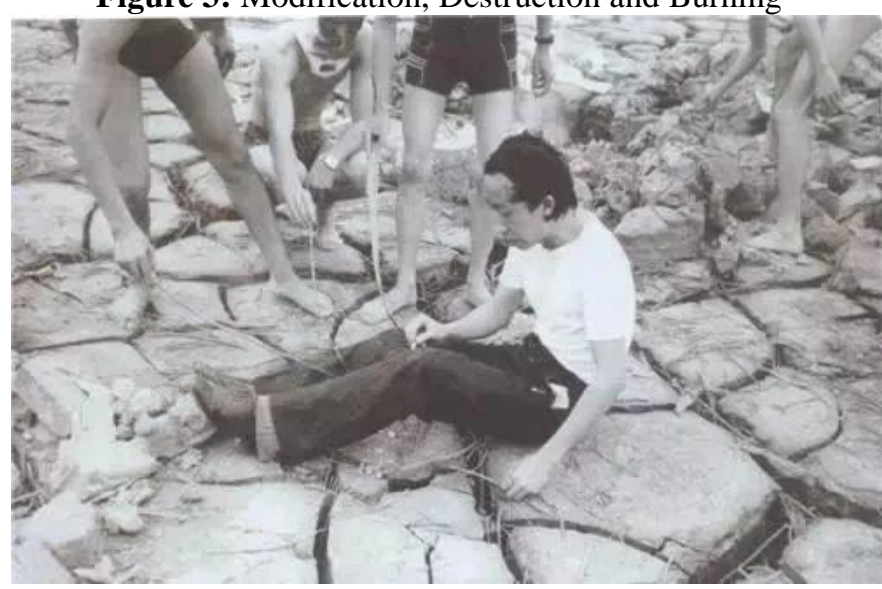

Figure 4: Firecracker Pants
Except "A Pistol Can Ring", the main bodies of the artworks above is performing art, and the main body of "a pistol that ring" is installation art. Not limited in performing art or installation art, the artworks of Xiamen Dada still adapted traditional painting approach. The reason that the author chose these 4 samples is that they are influential and image available. Meanwhile, performing art and Installation art were avant-guard at the time as well as they were in tune with Xiamen Dada's artistic proposition. It has therefore generated a great deal of interest in the society.

Lu Hong said that "the performing art and the installation art show the democracy principle in the art society through their interaction, daily and practical, they are always reactions against the mechanism of museum and gallery exhibition and collections in developed countries of Europe and America. They are the most direct mockery and combing of the capitalism market ideology under the collusion of power and capital." (Lu Hong, 2013) These two art forms represent Xiamen Dada's critique of the original art mainstream.

The study below has interpreted 4 artworks in Erwin Panofsky's image analysis, and add the analysis toward dynamic factors like performance, on the basis of the theory for image interpretation.

The earliest of these four works is "A Pistol Can ring", created in 1984 which is the time that between the "Five People Modern Painting Exhibition" and "Xiamen Dada Exhibition" This work consists of a bicycle bell, a lock and pipe to be an installation with a long tube and short shank. The bottom of the installation is the switch of the bicycle ring, and the lock is placed upside down under the pipe. Lock catch is located in the shank of the installation, while the pipe is the main body of this object which forms the shape of a pistol, with the lock serving as the handle of the pistol and a narrow water pipe replacing the barrel.

An installation with long tube and short shank expresses the conceptual pistol with its shape visually. While the pistol is a violent weapon which represents war and resistance, in intense atmosphere, the components that make up the pistol are replaced with everyday bicycle bell, lock and water pipe, and the juxtaposition of concepts forms a sense of contradiction. Meanwhile, as the topic of the artwork tells, "A Pistol Can Ring", the ringing moment of the pistol means the start of war and the moment of pain. However, this pistol can only make the sound of a bicycle bell ringing, which enhances the sense of conflict and contradiction. The resemblance of the sound and the shape intends to highlight the existence of the conflict, while the bell's ringing is the climax of the contradiction. The pistol shot, which symbolizes death and violence, and the ringing of the bicycle bell, which symbolizes the daily expression "Stay Away from the Car", express the same commanding response to the listener of the sound. In the meantime, they cause different result seperately.

"The Man Wrapped in Red Cloth and the Reef and Sea Sky", "Modification, Destruction and Burning" and "Firecracker Pants" both adapt the creative method of performing art. "The Man Wrapped in Red Cloth and the Reef and Sea Sky" was photographed at 1986 with three boys majored in fine art invited by Lin Chun. The photo quoted in this article is the 
most famous one among this series of photos. Overall, this photo shows that three trunks of people wrapped in red cloth, and the people in this photo are interacting with the reef by the seaside. An indescribable landscape is presented through the gestures of the hands and feet beyond the body of the red cloth. As for the "Modification, Destruction and Burning", it happened on 23 November 1986, which was after the "Xiamen Dada" Exhibition. The artists participated in the exhibition modified and destroyed their own artworks, and burnt them in the Xiamen New Art Square. These artworks perform in diverse format including painting, installation and so on. The artists wrote down the manifesto in the ground, "There is no peace in life without the elimination of art", "Dada is dead" and so on. "Firecracker Pants" was performed in 12 November 1987, the time that Xiamen Dada's members operated the "Entanglement-Bondage Activities". In the performance, Huang Yong-Ping placed firecrackers into his pants, and set the them alight and explode.

In each of these works, a variety of different symbols are involved. They each have a different meaning in terms of signifier, both "The Man Wrapped in Red Cloth and the Reef and Sea Sky" and "Firecracker Pants" deal with human bodies, the signified of them are close to "man" and "human life". In the work of Lin Chun, the man was wrapped in red cloth, but the red cloth cannot cover all their bodies, and their hands and feet are exposed. This scene obscured the specific characteristics of the person being covered, and created a sense of tension. The five fingers spread in an ambling gesture, as if in worship of some certain objects. The red color had special status in the cultural field. Firstly, it was the symbol of "Festive" and "Auspicious". Secondly, in the term of Cultural Revolution (1966-1976), "Red, Bright and Positive" is the outstanding model in the art of Cultural Revolution, the 'red' here symbolized advocating the socialism and proletarians in China. The red cloth covered the performers' bodies, this behavior can be interpreted in two approaches above, and these two approaches illustrated the conflict in the China society. Firecracker was the supplies for the ceremony. It was dangerous with explosion. Pants are the personal daily clothing, when firecrackers exploded in pants, this behavior enclose the distance of human and danger. As for "Modification, Destruction and Burning", the commonality of the three acts, the modification, the destruction and the burning of the work, lies in the dismantling of the original work, but to varying degrees. The modification gave a new and current meaning to the work, while the destruction devastated the value of works. The burning part completely Eliminated traces of the existence of the original works.

As for Iconographic analysis of above items. First of all, this phase of creation is reflective of the Cultural Revolution. The "Scar Literature" concept was transplanted to fine art field. Fine art works are also presented in the social and cultural sphere, as a means of exposing the problems of the Cultural Revolution. Although Xiamen Dada merged later than the presentation of above ideas, but their artworks still have a similar ideological character. This is because the members of Xiamen Dada share the same deep influence of the Cultural Revolution. For example, "A Pistol Can Ring" and "Firecracker Pants" both contained the intention to reflect on the violence of the Cultural Revolution. they also showed the alienation of things as violence invades everyday life, while "The Man Wrapped in Red Cloth and the Reef and the Sky and the Sea" showed people in a state of tension and confusion under the absolute 'red'.

Secondly, the creation of Xiamen Dada is a rebellion against the Cultural Revolution's art and the art form of the past. In the first place, its rebelliousness is reflected in its focus on people. After 10 years of the Cultural Revolution art, the absolute revolutionary and politicized art creation of "Three Prominences", "Red, Bright and Positive' and 'Tall broad-minded and Flawless", Lu Hong (2013). From 1976 to 1978, the artists started with the "life stream" style, 'The Serial Painting in Tibet' of Chen Dan-Qing and 'Water Festival-The Hymn to Life' of Yuan Yun-Sheng are outstanding masterpieces of this movement. "Man, and human's life" regained the spotlight of art creation in this period, it replaced the past model of art. Xiamen Dada was influenced by this wave and paid attention to the same theme of art.

While, it is presented in the art form, Lu Hong said that "if there is a common ground between advanced art and performing art, installation art, the commonality must be that they all aspire to 'revolution', to sabotage, to destroy, to rebel." It can be seen that the "rejection" of the easel painting form to a certain extent is in fact a rebellion against the artistic order and tradition in the art form.

Thirdly, Xiamen Dada is an essential part of "85 New Wave" which is important to Chinese contemporary history. The appearance of Xiamen Dada is relied on the critique toward "The Sixth National Art Exhibition of Excellent Works" organized by the Ministry of Culture and the National Art Association since October 1984. The critique directly led to rebellion and reflection in the art world at the time against the single style of art creation, the only single channel for art exhibitions and the determinism of subject matter. This critical force led directly to the outbreak of the "85 New Wave".

\section{Xiamen Dada: Birth and Dissolution}

This section analyses 11 letters represented by Lin Chun and Huang Yong Ping, and young art critics of the time, including Fan Di-An, Wang Xiao-Jian, Yan Shan-Chun and Gao Ming-Lu, in the 'Compilation of Historical Materials of the 85' Art Movement', compiled by Gao Ming-Lu. The letters sent by Huang Yong Ping are particularly numerous. In previous studies of Xiamen Dada, there has been little analysis of the correspondence between its members and the outside world. Correspondence are from January 1986 until February 1988, in the same time with Xiamen Dada's activities. The author classified these letters into three categories-letters seeking external support, letters discussing internal issues about the group and letters discussing the artworks. Some letters relate to more than one type, and are analysed in a corresponding perspective in the different categories. This paragraph will use the above analysis to restore the state of Xiamen Dada at each stage of its development, and the social context in which it was found. 


\subsection{The Letters Seeking External Support}

There are 5 letters seeking external support among 11 letters chosen for this paragraph. They are a letter from Lin Chun to Gao Ming-Lu dated 15 September 1989, a letter from Huang Yong-Ping to Fan Di-An dated 26 November 1986, a letter from Lin Chun to Gao Ming-Lu dated 12 December 1986, and letters from Huang Yong-Ping to Wang Xiao-Jian dated 16 February 1987 and 6 March 1987, respectively.

The first letter is Lin Chun informed Gao Ming-Lu, who stands for Art Magazine editorial board the issues about Xiamen Dada Exhibition, include the exhibition time, participated artists, the artworks and the site size, etc. The second letter was written at the same time of 'Modification, Destruction and Burning' performance which was after the Xiamen Dada Exhibition. There are 2 main purposes in this letter. The first one is asking Fan Di-An to write a critique for academical support toward the exhibition to express that "There is no boundary in artworks, unless we destroy it". The second purpose is preparing for the exhibition from May to June 1987 (which failed in that year). The third letter is the second correspondence between Lin Chun and Huang Yong-Ping, which had the similar purpose with the letter between Huang Yong-Ping and Fan Di-An. It included the images and textual descriptions of the two sessions of the exhibition, the modification of the works and the burning of the works, as well as they wished to publish the related records of conferences and Huang Yong-Ping's essay on the Art Magazine.

To summarize three letters above, the expectation of Huang Yong-Ping, Lin Chun and the other members of Xiamen Dada for further dissemination of their artworks and artistic concepts and for academic support. Meanwhile, the rejection of the mainstream of art is starting to appear. "Other publications won't work., just submit to you." At the same time, Lin Chun and Gao Ming Lu in his second correspondence also mentions Xiamen Dada's planned participation in a modern art exhibition planned for Beijing in May 1987, showing his vision to further and expand Xiamen Dada's influence.

The fourth and fifth letters differ from the above three letters in their eagerness to gain external support and further dissemination, but these two letters shown the dilemma faced by the Xiamen Dada group at that time, they were not accepted by the mainstream of art, and they lacked of channels through which content can be exported. Huang Yong-Ping said that 'we don't have a publication that belongs to our own'. As well Wang Xiao-Jian also served as a "martyr" to Xiamen Dada and even Chinese contemporary art at the time, for "supporting or advocating modern art and creating troubles for them". He was removed from his post.

\subsection{The Letters Discussing Internal Issues}

There are 4 letters discussing internal issues among 11 letters chosen for this paragraph. They are a letter from Huang Yong-Ping to Yan Shan-Chun dated January 1986, a letter from Huang Yong-Ping to Fan Di-An dated 26 November 1986, a letter from Huang Yong-Ping to Wang Xiao-Jian dated 19 October 1987 and a letter from Huang Yong-Ping to
Wang Xiao-Jian dated 19 February. These four letters were sent at the beginning, middle and end of Xiamen Dada's development, representing the different stages of Xiamen Dada's conceptual and future development.

The first letter was written after the Exhibition of Five Modern Painters, before the Xiamen Dada Exhibition. Huang Yong-Ping expressed his views on the 'confirmed image' that Yan Shan-Chun wanted to obtain in the letter. Huang Yong-Ping believes that "The uncertainty in artworks are also a kind of formats, which in line with my ideas of art creation, which so far I have yet to have a relatively stable idea, and perhaps the only stable is the Instability." This is the origin of the uncertainty in Xiamen Dada's art style, Huang Yong Ping did not intend to have a formal, stylized creation.

At the same time, the reply letter expresses Huang Yong Ping's view of art-art as an action that artificially links theoretical and non-theoretical acts. For Huang Yong Ping, innovation is only a quantitative and temporal thing, and does not lead to "qualitative" changes, even though many artists' innovations follow the expectations of society. Its contribution is limited.

The letter from Huang Yong-Ping to Fan Di-An has been described above. It indicated Huang Yong-Ping's consideration and rejection toward organizing art creation. He considered the exhibitions as "Non-converging gatherings" to seek "a kind of freedom and spontaneity". His perspective is consistent with his letter to Yan Shan-Chun, which is against formalized, deterministic artistic creation.

The third letter is written before the Entangled Movement, the last collective activity of Xiamen Dada. Huang Yong-Ping introduce the information about Xiamen Dada to Wang Xiao-Jian. Besides, Huang Yong-Ping began to 'think about something more personal', the disappearance of Xiamen Dada is evident in this stage. Meanwhile, He described his future art creative direction.

The forth letter is the last one of these letters, at the stage that Xiamen Dada has been quiet for so long. 'it is still and silent in 1987', however Huang Yong-Ping still desired to go to Beijing.

Four letters above demonstrated gradual clarification of Huang Yong-Ping's own creative ideas, showing his opposition to stylistic formality and determinism, which may also had contributed to his eventual tendency towards individuality and independence.

\subsection{The Letters Discussing the Artworks}

There are 2 letters discussing the artworks of Xiamen Dada among 11 letters chosen for this paragraph. They are a letter from Huang Yong-Ping to Gao Ming-Lu dated 22 August 1987, a letter from Lin Chun to Wang Xiao-Jian dated 27 November 1987. The former focuses on Huang Yong Ping's 1987 work The Great Carousel and classifies its 385 entries in eight categories; the latter describes 'The Man Wrapped in Red Cloth and the Reef and Sea Sky' by Lin Chun, it indicated his creative purpose and Lin Chun's future direction. 
Both letters were written during the latter stages of Xiamen Dada's activities, a period when the group had lost its ability to form a large-scale artistic activity and unable to gain support for dissemination. The fragmentation of the group also made it necessary for Lin Chun, Huang Yong Ping and others to focus on their own individual work.

\section{Xiamen Dada: Art without Essence}

This paragraph analyses the related official text and media materials in the 'Compilation of Historical Materials of the 85' Art Movement'. They demonstrated the changing relationship between the artistic mainstream and Xiamen Dada via the development process and art concepts of Xiamen Dada. This paragraph classified 11 text and media materials into 2 categories. The first category is completely written by Xiamen Dada, otherwise the other category is composed by the communication with people outside the group and comments written entirely by the outsiders. There are 7 materials in the first category which are came from Huang Yong-Ping mostly. The second category has 9 materials, 2 of them are communication, as well another 2 are reports and comments. While analyzing the materials, the authors of them will be the basis of the second classification. It systematically demonstrates the opinions of Xiamen Dada and the society at that time.

\subsection{The Text Materials Written by Xiamen Dada's Members}

There are 7 materials of Xiamen Dada's members, mostly written by Huang Yong-Ping. 3 of them are the declarations or pretexts of the exhibitions and art activities. 5 of them deal with the study of theories and concepts related to Dada.

Huang Yong-Ping published the "Xiamen Dada: A Kind of Post-Modern?" in the "Chinese Art Report", This was the first article of Huang Yong-Ping that related to Xiamen Dada, combined the function of theoretical discussion and the function of activity declaration. It summarized the art activity happened from 1983 to 1986 in China, described the social background when Xiamen Dada appeared in China. "A postmodern period that is permeable, participatory, compatible, and inclusive of life (including pre-modernism and all its opposites) in its plurality has arrived"; "The time to explicitly present the spirit of 'Dada' in China seems to have arrived." Huang Yong-Ping connected the Chinese classical philosophy of "Zen" and "Dada", this article can be considered as the claim of Xiamen Dada's appearance, the theoretical study of the post-modernism wave in China. As well it's one of the significant symbols of Xiamen Dada's happening.

After that, "The Claim of Burning the Xiamen Dada" written by Huang Yong-Ping is called as the declaration of the performing art "Modification, Destruction and Burning". The statement of the exhibition of events that took place in the Fujian Fine Arts Exhibition Hall indicated the purpose of the activity which is the breakdown of the view of "art", "the sudden influx of these things into the museum has a certain attack, not on the visitor, but on the visitor's view of 'art'". In this declaration, "It is unfortunate that museums will take for granted that these things are works of art, as is the conventional wisdom" This sentence demonstrated the rebellion of Xiamen Dada toward the museum system, though it is covered in this occasion which is the resistance of the "Art Mainstream".

In 1987, Huang Yong-Ping published "Huang Yong-Ping's autobiography", "Theory against theory" and "The Art, An Ominous Object". In 1988, he published "The Completely Empty signans"-"Dada" and "Zen", which expresses the views of the work itself and Huang Yong-Ping's own theoretical and philosophical views on Dada, as well as Dada's skeptical and rebellious spirit. "The Art, An Ominous Object" and "The Completely Empty Noumenon"-"Dada" and "Zen" had strong theoretical features. They represented the link between Huang Yong Ping and "Dada". The former one indicated Huang Yong-Ping's doubt to the art, art history and even the social representative of artists. He was against to the leading force of the "potential model", "invent something that didn't exist before". Also, he considered that "My personal subjective will inevitably participate in this world". We have to adopt a "must not" attitude, use it with serenity, and deal with its "result" either with "sorrow and 'tears' or with 'epithets"". This theoretical Characteristics can also be seen on his creation. The latter analyzes the word "dada" through Saussure's semiotic theory and Roland Barthes' mythological theory, and argues that "dada" is completely empty signans, which is the first time he analyzes the semiotic meaning of the word "dada".

\subsection{The Communication and the Comments}

Among the written materials recorded in Gao Minglu's book, there are four pieces of materials and related comments related to Xiamen Dada, written by members outside the group or by members outside the group together with Xiamen Dada members. They are Essays on the Xiamen Dada "11-23 Art Event" written by Chen Yongpeng in connection with the event that took place on November 12, 1986, "Dialogue on Modern Art", a conversation between Fan Di-An and Huang Yong-Ping in February 1987, "Entangled Movement" written by Wu Yi-Ming in 1987, and "Summary of the November 30, 1987 Discussion" written by Lin Chun with the participation of Xiamen Dada members and students. "Entangled Movement" written by Wu Yi-Ming in 1987, and "Minutes of the November 30, 1987 Discussion" written by Lin Chun, a member of Xiamen Dada. Among them, Essays on Xiamen Dada's "11-23 Art Event" and "Entangled Movement" are commentaries and reports, respectively, documenting the "11-23" event the "modification- destruction-burning" event and its shocking effects-"The fire of the '11-23' event has transformed the art from the canvas, but also from the exhibits and galleries." The "Entangled Movement" event is a literal news report of the eponymous event, describing it and documenting the intentions of the organizers. The participators considered the event straightforward, without any external interpretation of meaning. It's a "form of meaninglessness", a report that makes up for the lack of official textual description of the event, and indicated that Dada continues to question the boundaries and meaning of "art". While the "Dialogue on Modern Art" and "Summary of the November 30, 1987 Discussion" are the documented articles of the conversation and forum. The former records the discussion between Fan Di-An and Huang Yong-Ping in 
February 1987 about the spiritual essence of "our culture" and the "mission of art", after a series of artistic practices, Huang Yong-Ping's questioning of art rose to the level of culture. Huang Yong Ping's questioning of art has risen to the level of culture. The latter is a record of a lecture given by members of Xiamen Dada at the invitation of the Fine Arts Department of Xiamen University, in which students questioned Dada members about the Dada exhibition itself and the issue of artistic standards. The lecture has shown the nihilistic nature of Xiamen Dada.

\section{Conclusion}

Through the study of Xiamen Dada's works, letters and documents, this paper restores the concrete and microscopic reality of Xiamen Dada, making up for the lack of research on the content of the group itself in previous studies.

In the first part of the analysis, the works of Xiamen Dada are mostly related to the conflicts in the social environment of the time. During "the Cultural Revolution", political power violently invaded everyday life, and the extremist, template art form isolated the spread of news concepts and culture. A series of opposing forces, such as new and old, confinement and freedom, fixation and emptiness, are naturally presented in Xiamen Dada's works.

In the second part of the analysis of the letters, the correspondence between Xiamen Dada members and the young critics shows the change in their mentality from the beginning of their activities to the time of their dissolution. Instead of disappearing, Xiamen Dada's silence became a source of sustenance that continues to influence the history of contemporary Chinese art.

The analysis of documents in the third section restores the relevance of Xiamen Dada's theory and practice from an objective perspective, and composes it in a chronological perspective. Huang Yong-Ping extended the view that "art has no essence" to art-related activities such as art creation, art group assemblages, and even exhibition systems.

As a product of the social and cultural environment, Xiamen Dada not only incorporates the critical features of postmodern philosophical thinking, but also has a long-lasting impact on the subsequent process of Chinese contemporary art with its radical disruptive and rebellious nature.

\section{References}

[1] Fei Da-Wei, '85 New Wave Documentaries, Xiamen Dada, Huang Yong-Ping, (Shanghai People Publication, 2007)

[2] Lu Ming Jun, Dada in The History: Art and Politics (Rong Bao Zhai, 2010)

[3] Zhang Si-Qi, The Research toward Huang Yong-Ping's Art Ideas (Nanjing Art Academy, 2019)

[4] Shao Tian-Hua, The Reflection to the Art of 'Xiamen Dada', (Journal of Xiangnan University, 2013)

[5] Li Sheng-Zhao, "Xiamen Dada" and the History of Ideas behind it (China Academy of Art, 2010.)

[6] Lu Hong, 30 Years of Chinese Contemporary Art: 1978-2008 (Hunan Fine Arts Publishing, 2013), P.3.
[7] Gao Ming-Lu, 85 Art Movement: A Compilation of Historical Information (Guangxi Normal University Press, 2007), p509-p541 